\title{
Modulation in InAs Quantum Dot Waveguides
}

\author{
B. Imran Akca ${ }^{1}$, Aykutlu Dana ${ }^{2}$, Atilla Aydinli ${ }^{2}$, Marco Rossetti ${ }^{3}$, Lianhe $\mathrm{Li}^{3}$, Andrea Fiore ${ }^{3}$, N. Dagli ${ }^{4}$ \\ Material Science and Nanotechnology Program, Bilkent University, Ankara, Turkey ${ }^{I}$ \\ Physics Department, Bilkent University, Ankara, Turkey ${ }^{2}$ \\ Institute of Quantum Electronics and Photonics, Ecole Polytechnique Fédérale de Lausanne EPFL, \\ CH-1015 Lausanne, Switzerland ${ }^{3}$ \\ ECE Department, University of California at Santa Barbara, California, USA ${ }^{4}$ \\ 'imran@bilkent.edu.tr
}

\begin{abstract}
Modulation in molecular beam epitaxy grown self-assembled InAs quantum dot waveguides have been studied at $1500 \mathrm{~nm}$ as a function of wavelength and voltage. Enhanced electro-optic coefficients compared to bulk GaAs were observed.

(C)2007 Optical Society of America

OCIS codes: (230.4110) Modulators; (250.7360) Waveguide modulators
\end{abstract}

Nonlinear optical and linear electro-optic materials find use as switching and modulation devices in photonic integrated circuits. Quantum dots and quantum wells have recently attracted much attention due to their enhanced electro-optic effects. Low dimensional quantum confined structures are expected to significantly enhance the performance of various optoelectronic components. [1] In this regard, we study optical transmission through multilayer InAs quantum dot lasers to investigate the degree of enhancement in electro-optic coefficient.

We worked on two different types of quantum dot structures, with three (3QDs) and five layers (5QDs) of quantum dots. The waveguides with 3QDs are in the lengths of 1,1.3,1.6 $\mathrm{mm}$ and the 5QDs waveguides are 1 and $2.3 \mathrm{~mm}$. The samples are grown by molecular beam epitaxy. The active region is formed by three or five layers of self-assembled InAs QDs, which are covered by a 5- $\mathrm{nm} \mathrm{In}_{0.15} \mathrm{Ga}_{0.85}$ As QW and separated from each other by a 40$\mathrm{nm}$ GaAs spacer layer. The areal dot density of our lens-shaped QDs is $3 \times 10^{10} \mathrm{~cm}^{-2}$. The active region is clad by 1.5 $\mu \mathrm{m}$ of $\mathrm{Al}_{0.7} \mathrm{Ga}_{0.3} \mathrm{As}$, which is $n$-doped on the substrate side and $p$-doped on the top side. Our devices also work as ridge-waveguide lasers with 8 and $15 \mu \mathrm{m}$ widths. Both facets are as cleaved. For each sample lasing is peaked at nearly $1285 \mathrm{~nm}[2]$.

Measurement of the electro-optic coefficients away from EL emission peak was carried out by coupling $1.5 \mu \mathrm{m}$ light from a tunable laser (Santec Tunable LD Light Source TSL-520) onto one end of the waveguide with a lens shaped fiber. A controlled DC voltage source was used to apply 0 to 6 Volts reverse bias to the samples. At each voltage level, the transmission through the device is recorded as a function of wavelength. The index of refraction can then be inferred from the Fabry-Perot cavity response. The experimental results and their optimum curve fits are given in Fig. 1 and $\mathbf{2}$.
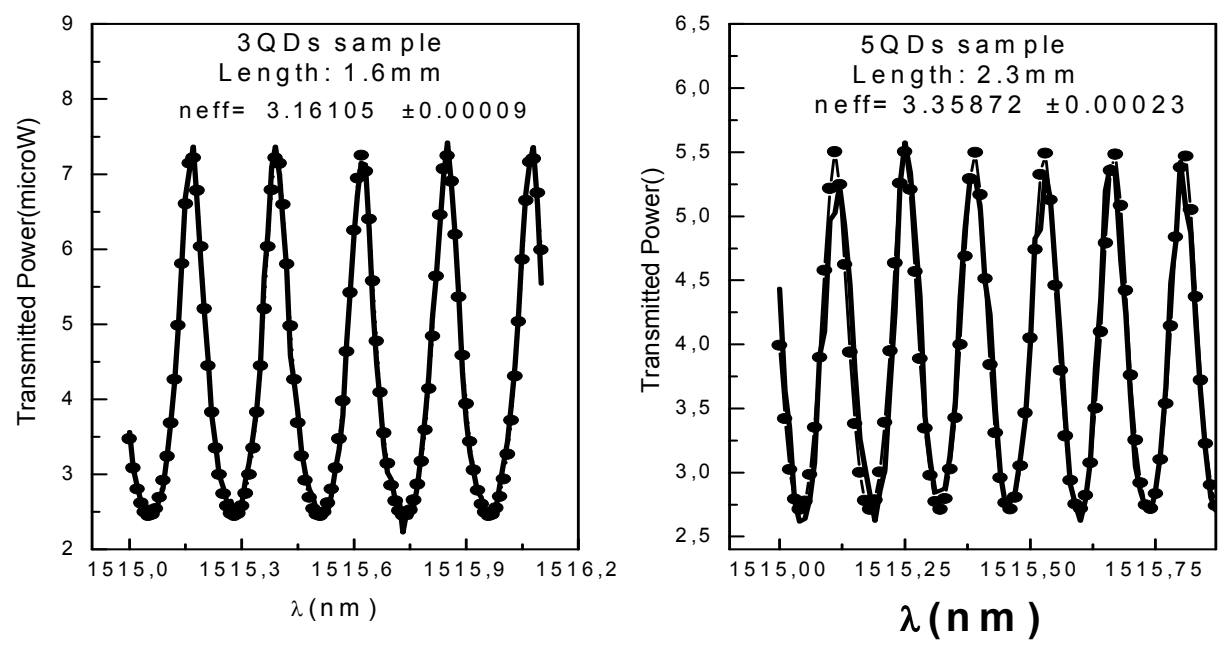

Figure 1. (a) The transmission power versus wavelength of the 5QDs sample. The circular dots show the optimum curve fit and the other curve is the representation of the experimental data at zero volt. (b) Same graphs for 3QDs sample. 


\section{IWE4.pdf}
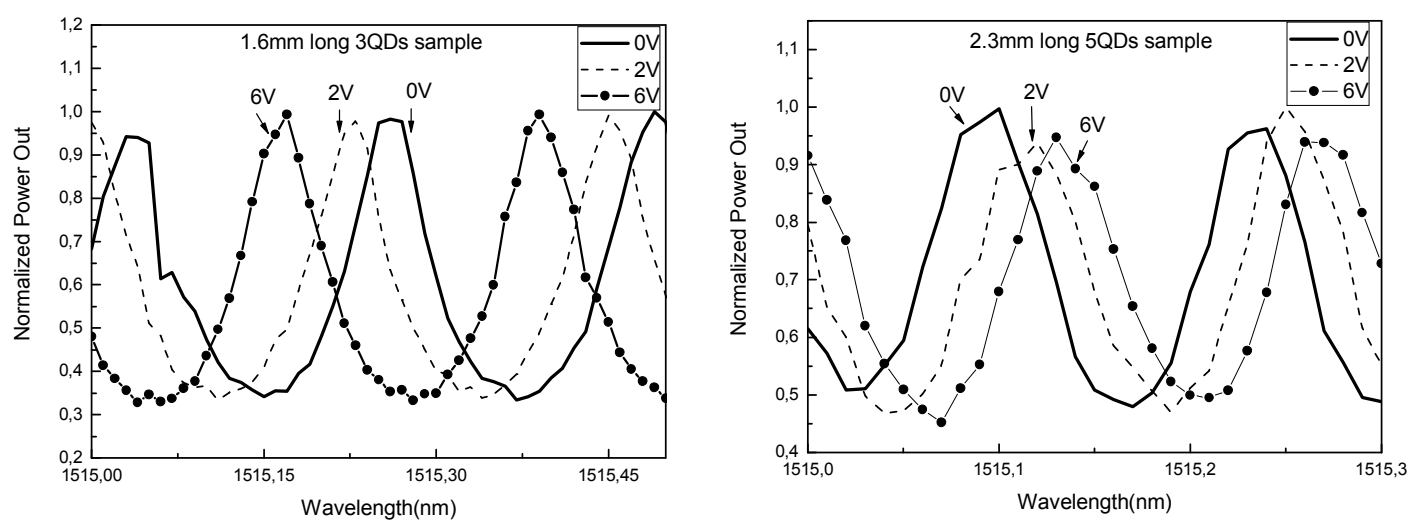

Figure 2. Transmission of the Fabry-Perot modulator as a function of the wavelength for 3QDs and 5QDs structures. Significant tuning is observed with relatively low voltages.

The linear electro-optic coefficients are obtained by fitting the measured data to the equation of transmission through a Fabry Perot modulator [3] and calculating the refractive index difference between two specific voltages. The change in refractive index due to applied voltage is $\Delta n(V)$ is given as [3]

$$
\Delta n(V)=\frac{1}{2} n_{e}^{3} r_{41} \frac{V}{t} \Gamma
$$

where $r_{41}$ is the electro-optic coefficient, $t$ is the thickness of the epilayer ( $\mathrm{t}=135 \mathrm{~nm}$ for 3QDs sample and $\mathrm{t}=225 \mathrm{~nm}$ for 5QDs sample), $n_{e}$ is the effective index, $V$ is the voltage difference between two curves and confinement factor $\Gamma$ is the overlap of the vertical electric field component with the optical mode. To obtain $\mathrm{r}_{41}, \Gamma$ needs to be known precisely. It is clear that the confinement factor is a combination of the individual confinement factors of each material in the active region of the waveguide. Each layer contributes a different amount to the mode confinement, roughly proportional to their thickness, and quantum dot layers have the lowest confinement factor compared to GaAs and InGaAs layers due to their small fill factor in the active region. For mode shape calculations, the effect of the quantum dots on confinement due to bulk refractive index difference is neglected; we only considered the contributions of the other layers. This approximation ensures that a conservative estimate of the electro-optic coefficient is made. We, therefore present $r_{41}$ normalized to $\Gamma$. The results of the measurements are given in Table 1 .

Table1. Measurement results for 3QDs and 5QDs samples.

\begin{tabular}{|l|c|l|l|c|}
\hline \multicolumn{1}{|c|}{$\begin{array}{c}\text { Type of } \\
\text { sample }\end{array}$} & \multicolumn{3}{|c|}{$\begin{array}{c}\text { Refractive index } \\
\text { (Voltage dependent) }\end{array}$} & $\begin{array}{c}\mathrm{r}_{41} / \Gamma \\
\left(\times 10^{-13} \mathrm{~m} / \mathrm{V}\right)\end{array}$ \\
\hline & $0 \mathrm{~V}$ & \multicolumn{1}{|c|}{$2 \mathrm{~V}$} & $6 \mathrm{~V}$ & \\
\hline 3QDs -1.6mm & 3.16123 & 3.16119 & 3.16105 & 7.7 \\
\hline 3QDs -1.3mm & 3.16829 & 3.16866 (for 3V) & 3.16858 & 6,8 \\
\hline 3QDs -1mm & 3.16661 & 3.16762 (for 3V) & 3.16687 & 5 \\
\hline 5QDs -2.3mm & 3.35862 & 3.35872 & 3.35904 & 17 \\
\hline 5QDs -1mm & 3.39115 & 3.39728 & 3.39813 & 13 \\
\hline
\end{tabular}

These values are considerably higher than that of the materials surrounding the QDs. As a matter of fact examination of Figure 2 reveals that full on/off modulation is possible for $1.6 \mathrm{~mm}$ long 3 QD sample using $6 \mathrm{~V}$. This corresponds to less than $1 \mathrm{~V}-\mathrm{cm}$ modulation efficiency. In other words using this modulator as the arms of a push pull driven Mach-Zehnder modulator, less than $1 \mathrm{~V}$ drive voltage would result for $1 \mathrm{~cm}$ long arms. This should be possible since the propagation loss based on the fringe visibility is less than $5 \mathrm{~dB} / \mathrm{cm}$. This is far more efficient than what can be obtained using bulk materials without the QDs. 


\section{IWE4.pdf}

In conclusion, we have observed the low voltage modulation in InAs quantum dot waveguides. We have obtained the electro-optic coefficients for several waveguides with different lengths and layers of InAs quantum dots. Electro-optic coefficients as large as $7.7 \times 10^{-13} / \Gamma(\mathrm{m} / \mathrm{V})$ for 3 QDs samples and $17.0 \times 10^{-13} / \Gamma(\mathrm{m} / \mathrm{V})$ for the 5 QDs samples were obtained. This is an enhancement in both cases compared to the bulk GaAs [1].

Acknowledgement: This project is supported by TUBITAK; Grant No: 103 T115.

\section{References:}

[1]. L. Davis, K. Ko, W. Li, H.Sun, Y. Lam, T. Brock, S. Pang, and P. Bhattacharya, "Photoluminescence and electro-optic properties (25-35 nm diameter) quantum boxes", Appl. Phys. Lett., vol. 62, No.22, May 1993

[2]. A. Markus, J. X. Chen, C. Paranthoen, and A. Fiore," Simultaneous two-state lasing in quantum-dot lasers," Applied Physics Letter, vol. 82, No. 12, March 2003.

[3]. S. Nishimura, H. Inoue, H. Sano, and K. Ishida "Electrooptic Effects in an InGaAs/InAlAs Multiquantum Well Structure," IEEE Photonics Technology Letters, vol. 4, No. 10, October 1992.

[4]. Yariv and P. Yeh, "OpticaI Waves in Crystals", (Wiley, New York, 1984). 